\title{
SURVEY OF ROOF RAT Rattus rattus, (Linn.) IN EL-IBRAHEMIA DISTRICT, SHARKIA GOVERNORATE
}

\author{
HEGAB, A. M. I. ${ }^{1}$, A. A. F. EL-BAHRAWY ${ }^{2}$, G. A. EL-KADY ${ }^{2}$ AND \\ M. I. A. ELBKHSHWANGI ${ }^{1}$
}

1. Plant Protection Research Institute, $A R C$, Dokki, Giza

2. Dept. of Plant Protection, Faculty of Agriculture, Suez Canal University, Egypt

(Manuscript received 8 February 2009)

\begin{abstract}
Survey of the roof rat Rattus rattus (Linn.) by live traps in the three different locations, (houses, granaries and poultry farm) in El-Ibrahemia district, Sharkia Governorate were studied, during two successive years (2004/2005 and 2005/2006). The total number of $R$. rattus (Linn.) were (435 and 329 individuals), (84 and 67 individuals) and (86 and 66 individuals) recorded in houses, granaries and poultry farm during the $1^{\text {st }}$ and $2^{\text {nd }}$ years, respectively. The data indicated that females outnumbered males all over the year in the three studied locations except during the $2^{\text {nd }}$ year, in the granaries. The number of mature stages exceeded that of the immature ones at the different locations during all the studied years. On the other hand, the poultry farm obtained the highest average number of embryos per pregnant female for the roof rat was 7.1 and 7.3 during the $1^{\text {st }}$ and $2^{\text {nd }}$ years, respectively.

Keywords: Rattus rattus (Linn.) - Sex ratio - Maturity status - Reproduction.
\end{abstract}

\section{INTRODUCTION}

Wild rats can attack all kinds of cereal crops, vegetables, fruits, granaries, stored food and grains. World wide, an annual loss of $3.55 \%$ of stored grains is to the damage of $R$. rattus Dykstra (1966) and rat damage occurs during all phases of food production and storage. Rodent's problem increased in the last two decades. The main reasons are due to the diversion in agricultural system and construction of new cities in the desert areas and the wide usage of pesticides for controlling agricultural pests that leads to the kill of natural enemies of rodents i.e. reptiles and wild birds.

In Egypt, several authors studied the sex ratio, maturity status on rodents in a semi desert area (Arafa, 1968, Abdel-Gawad et al., 1982, El-Bahrawy, 1986, Abd-ElKarim, 1991, Youssef, 1996, Embarak, 1997, Hegab, 2004, Hegab et al., 2006 and ElBahrawy et al., 2008).

The aim of the present work is to estimated data of the trapped rats: sex ratio, maturity status, number of pregnant, non pregnant females and number of embryos in 
uterus per each pregnant female for the roof rat (Rattus rattus) in the three different locations at El-Ibrahemia district, Sharkia Governorate.

\section{MATERIALS AND METHODS}

The present study was carried out in three different locations, houses, granaries and poultry farm at El-Ibrahemia district, Sharkia Governorate during two successive years (2004/2005 and 2005/2006). Study started from November to the end of October for the next year. The first location was urban area including many houses (100 houses), the second location was cereal granaries (5 granaries), with an air opening storage containing cereal grains such as unhusked rice, rice and wheat. The granaries were surrounded by numerous houses and water canal. The third location was a poultry farm it is about area $300 \mathrm{~m}^{2}$ and consists of two floors building and a feed storage.

Rats were trapped alive using wire-box traps with spring doors, traps were cleaned by water and liquid soap before each use. The traps were supplied with fresh bait materials, namely: tomato slices, bread pieces and fired fish. In each investigated location forty wire-box traps were baited with fresh bait and distributed every two week in different positions. In the houses the traps were placed in the infested sites. In the granaries the traps were placed beside the walls at distances of $10 \mathrm{~m}$ in between. In the poultry farm the traps were placed in the feed storage and inside the farm beside the wall. The traps were left from 6 p.m. at the above mentioned locations and collected in the morning at 7 a.m. Positive traps containing rats were separately enclosed in white cloth bags fastened with a string and transferred carefully in the same day to the laboratory for examination, El-Bahrawy et al. (2008).

The trapped rats were individually inserted into a killing jar provided with a piece of cotton wool moistened with chlorophorm for anesthetizing rats.

The estimated datafrom the trapped rats were:

1- Identification and classification into species according to the Osbron and Helmy (1980).

2- The density, sex ratio and maturity.

3- Each trapped female rats weighted and dissected to measure:

A- Number of pregnant and non pregnant females.

B- Number of embryos per each pregnant female. 


\section{RESULTS AND DISCUSSION}

\section{Population density, sex ratio and maturity status of the roof rats}

\subsection{In houses}

Results in Tables (1\&2) indicated that the total numbers of the roof rat $R$. rattus (Linn.) were 435 and 329 individuals recorded during the $1^{\text {st }}$ year 2004/2005 and the $2^{\text {nd }}$ years 2005/2006, respectively. The highest number of $R$. rattus (Linn.) was 68 and 56 individuals recorded during August through $1^{\text {st }}$ and $2^{\text {nd }}$ years, respectively. While lowest number was 12 and 9 individuals recorded during March through $1^{\text {st }}$ and $2^{\text {nd }}$ years, respectively. The sex ratio (male/female) was 0.91:1.09 and 0.98:1.02 during the $1^{\text {st }}$ and $2^{\text {nd }}$ years, respectively. The maximum population density for male and female of the roof rats was ( 31 and 28 individuals) and (37 and 28 individuals) recorded during August 2005 and 2006, respectively.

On the other hand, the high maturity numbers of males were 15 and 21 individuals recorded during April, May, August and September 2005 and June 2006, respectively, while the highest immature numbers of males were 16 and 15 individuals recorded during August 2005 and 2006, respectively. It appears also that the highest numbers of mature females was 26 and 18 individuals recorded during May 2005 and August 2006, respectively. The maximum numbers of immature females were 18 and 12 individuals observed during August 2005and 2006, respectively. The population of mature stages exceeded that of the immature ones all the two years recording average of (65.7 and $34.3 \%)$ during the $1^{\text {st }}$ year, $(72.6$ and $27.4 \%)$ during the $2^{\text {nd }}$ year, respectively. These data agree with those obtained by Gaaboub et al. (1978) who concluded that, spring and summer represent the main reproduction season of the rat species in the surrounding area of Alexandria Governorate. Embarak (1997) observed that, the highest density of immature roof rats was reached in summer and spring in cultivated and newly reclaimed areas in Assiut Governorate, respectively.

\subsection{In granaries}

Data presented in Tables (3\&4) showed that the total number of the roof rats $R$. rattus (Linn.) were 84 and 67 individuals recorded during the $1^{\text {st }}$ and $2^{\text {nd }}$ years, respectively. The highest number of $R$. rattus (Linn.) was 20 and 13 individuals recorded during May and November through $1^{\text {st }}$ and $2^{\text {nd }}$ years, respectively. While lowest number was 1 and 2 individuals recorded during June and April through $1^{\text {st }}$ and $2^{\text {nd }}$ years, respectively. The sex ratio for male to female was 0.95:1.05 and 1.37:0.63 during the $1^{\text {st }}$ and $2^{\text {nd }}$ years, respectively. The highest numbers of females were 9 and 5 individuals recorded during April and November through $1^{\text {st }}$ and $2^{\text {nd }}$ years, respectively 
The average numbers of mature stage for males increased from 30 individuals (75\%) and 44 individuals (95.7\%) during 2004/2005 and 2005/2006, respectively. Also, the average numbers of mature stage for females were 40 individuals (90.9\%) and 13 individuals $(61.9 \%)$ during the $1^{\text {st }}$ and $2^{\text {nd }}$ years, respectively. The average numbers of mature stages were 70 individuals (83.3\%) and 57 individuals (85.1\%) during the $1^{\text {st }}$ and $2^{\text {nd }}$ years, respectively, while it was 14 individuals $(16.7 \%)$ and 10 individuals $(14.9 \%)$ for immature stages during the $1^{\text {st }}$ and $2^{\text {nd }}$ years, respectively. These results are in harmony with those obtained by Abdel-Gawad (1974 and 1979) who reported that, females outnumbered males. The highest density of immature roof rat was reached in autumn. The mature was surpassed immature stage in all seasons in the cultivated area at Assiut Governorate. El-Bahrawy (1986) found that, the mature stages outnumbered immature ones, being $81.85 \%$ mature and $18.15 \%$ immature for R. r. frugivorus in Ismailia Governorate. Abd-El-Karim (1991) who found that, the population of mature stage of $R . r$. frugivorus and $R . r$. alexandrinus exceeded that of the immature ones all the year round.

\subsection{In poultry farm}

Tables (5\&6) showed that the total numbers of the roof rats were 86 and 66 individuals recorded during the $1^{\text {st }}$ and $2^{\text {nd }}$ years, respectively. The highest number of $R$. rattus (Linn.) was 15 and 17 individuals recorded during May through $1^{\text {st }}$ and $2^{\text {nd }}$ years, respectively. While lowest number was 2 and 1 individuals recorded during July through $1^{\text {st }}$ and $2^{\text {nd }}$ years, respectively. The sex ratio (male/female) was 0.81:1.19 during the $1^{\text {st }}$ year and 0.88:1.12 during the $2^{\text {nd }}$ year.

It appears also that the average of mature stage for males recorded $62.9 \%$ during $2004 / 2005$ and $62.1 \%$ during $2005 / 2006$. On the other hand, the averages of mature stage for females were 84.3 and $48.6 \%$ during the $1^{\text {st }}$ and $2^{\text {nd }}$ years, respectively. In general, mature stage outnumbered immature ones recording an average of $24.4 \%$ (immature) and $75.6 \%$ (mature) during the $1^{\text {st }}$ year, while $45.5 \%$ (immature) and $54.5 \%$ (mature) during the $2^{\text {nd }}$ year. It is clear also from the data that, the roof rats were not observed during the period from August till October 2006. Most of poultry farms are destroyed by virus Fowel infelwanza (H5N2) during this stage. These results agree with those obtained by Omar (1977) who mentioned that, statistical analysis indicated no-significant difference between the numbers of females and males of immature and mature stages of $R$. r. alexandrinus. Mourad et al. (1982) reported that, the highest density of $R$. rattus (linn.) was noticed during spring season followed by autumn and the lowest density was during winter and summer seasons. 


\section{Numbers of embryos in pregnant females}

Results in Tables (7\&8) showed that the average numbers of embryos per pregnant females in houses, granaries and poultry farms were (6.8 and 6.7), (6.9 and 6.0) and (7.1 and 7.3) during the $1^{\text {st }}$ and $2^{\text {nd }}$ years, respectively. The total numbers of non pregnant females for $R$. rattus (linn.) were 142 and 115 individuals during the $1^{\text {st }}$ and $2^{\text {nd }}$ years, respectively, but the total numbers of pregnant females were 96 and 36 individuals during the $1^{\text {st }}$ and $2^{\text {nd }}$ years, respectively. The highest numbers of non pregnant females were 25 and 17 individuals recorded during May 2005 and August 2006, respectively. While, the highest numbers of pregnant females were 17 and 8 individuals recorded during May 2005 and 2006, respectively.

On the other hand, the highest average numbers of embryos 8.0 for three locations were observed during four months November 2004, (February and March 2005) and January 2006. The lowest average numbers of embryos were 5.6 and 5.0 recorded during January 2005 and March 2006, respectively. While no recorded embryos during February 2006. In the houses, the highest average numbers of embryos for the roof rats $R$. rattus (linn.) were obtained during March 2005 (11.0) and January 2006 (8.0), but in the granaries were 8.0 and 6.0 during May 2005 and 2006, respectively. While, the highest average numbers of embryos were 9.5 and 8.0 during September 2005 and May 2006 in the poultry farm, respectively. The total numbers of embryos in poultry farm more than houses and granaries because females fed on foods, eggs and bodies of dead poultry which are rich source of hormones, proteins and fatty acids which may be stimulate reproduction activity in $R$. rattus (linn.). These results agree with those obtained by Abd-El-Karim (1991) who stated that, the average number of embryos per female for $R$. $r$. frugivorus was 6.99 with maximum of 7.6 during May and minimum of 6.2 embryos per female during August. Embarak (1997) found that the mean number of embryos per captured females from cultivated area during the first one year was recorded to be 6.76 and 7.00 , while during the second year was found to be 8.97 and 6.70 for $R$. r. frugivorus and $R$. r. alexandrinus, respectively. 
Table 1. Number of trapped roof rats Rattus rattus (Linn.), their sex ratio and maturity status at the houses location during year of $2004 / 2005$.

Sex ratio (males/females) $=0.91: 1.09$

\begin{tabular}{|c|c|c|c|c|c|c|c|c|c|c|c|c|c|c|c|c|c|}
\hline \multirow{4}{*}{ Months } & \multirow{4}{*}{$\begin{array}{l}\text { No. of } \\
\text { Rats }\end{array}$} & \multicolumn{4}{|c|}{ Sex } & \multicolumn{12}{|c|}{ Maturity status } \\
\hline & & \multirow{2}{*}{\multicolumn{2}{|c|}{ Males }} & \multirow{2}{*}{\multicolumn{2}{|c|}{ Females }} & \multicolumn{4}{|c|}{ Males } & \multicolumn{4}{|c|}{ Females } & \multicolumn{4}{|c|}{ Total } \\
\hline & & & & & & \multicolumn{2}{|c|}{ Immature } & \multicolumn{2}{|c|}{ Mature } & \multicolumn{2}{|c|}{ Immature } & \multicolumn{2}{|c|}{ Mature } & \multicolumn{2}{|c|}{ Immature } & \multicolumn{2}{|c|}{ Mature } \\
\hline & & No. & $\%$ & No. & $\%$ & No. & $\%$ & No. & $\%$ & No. & $\%$ & No. & $\%$ & No. & $\%$ & No. & $\%$ \\
\hline Nov.04 & 14 & 7 & 50.0 & 7 & 50.0 & 2 & 28.6 & 5 & 71.4 & 2 & 28.6 & 5 & 71.4 & 4 & 28.6 & 10 & 71.4 \\
\hline Dec. & 13 & 6 & 46.2 & 7 & 53.8 & 3 & 50.0 & 3 & 50.0 & 2 & 28.6 & 5 & 71.4 & 5 & 38.5 & 8 & 61.5 \\
\hline Jan.05 & 19 & 11 & 57.9 & 8 & 42.1 & 0 & 0.0 & 11 & 100.0 & 1 & 12.5 & 7 & 87.5 & 1 & 5.3 & 18 & 94.7 \\
\hline Feb. & 26 & 11 & 42.3 & 15 & 57.7 & 0 & 0.0 & 11 & 100.0 & 2 & 13.3 & 13 & 86.7 & 2 & 7.7 & 24 & 92.3 \\
\hline Mar. & 12 & 6 & 50.0 & 6 & 50.0 & 0 & 0.0 & 6 & 100.0 & 3 & 50.0 & 3 & 50.0 & 3 & 25.0 & 9 & 75.0 \\
\hline Apr. & 51 & 20 & 39.2 & 31 & 60.8 & 5 & 25.0 & 15 & 75.0 & 8 & 25.8 & 23 & 74.2 & 13 & 25.5 & 38 & 74.5 \\
\hline May & 57 & 23 & 40.4 & 34 & 59.6 & 8 & 34.8 & 15 & 65.2 & 8 & 23.5 & 26 & 76.5 & 16 & 28.1 & 41 & 71.9 \\
\hline June & 49 & 20 & 40.8 & 29 & 59.2 & 8 & 40.0 & 12 & 60.0 & 12 & 41.4 & 17 & 58.6 & 20 & 40.8 & 29 & 59.2 \\
\hline July & 39 & 19 & 48.7 & 20 & 51.3 & 6 & 31.6 & 13 & 68.4 & 10 & 50.0 & 10 & 50.0 & 16 & 41.0 & 23 & 59.0 \\
\hline Aug & 68 & 31 & 45.6 & 37 & 54.4 & 16 & 51.6 & 15 & 48.4 & 18 & 48.6 & 19 & 51.4 & 34 & 50.0 & 34 & 50.0 \\
\hline Sep. & 52 & 25 & 48.1 & 27 & 51.9 & 10 & 40.0 & 15 & 60.0 & 9 & 33.3 & 18 & 66.7 & 19 & 36.5 & 33 & 63.5 \\
\hline Oct. & 35 & 18 & 51.4 & 17 & 48.6 & 9 & 50.0 & 9 & 50.0 & 7 & 41.2 & 10 & 58.8 & 16 & 45.7 & 19 & 54.3 \\
\hline Total & 435 & 197 & - & 238 & - & 67 & - & 130 & - & 82 & - & 156 & - & 149 & - & 286 & - \\
\hline$\%$ & - & - & 45.3 & - & 54.7 & - & 34.0 & - & 66.0 & - & 34.5 & - & 65.5 & - & 34.3 & - & 65.7 \\
\hline
\end{tabular}


Table 2. Number of trapped roof rats Rattus rattus (Linn.), their sex ratio and maturity status at the houses location during year of 2005/2006.

\begin{tabular}{|c|c|c|c|c|c|c|c|c|c|c|c|c|c|c|c|c|c|}
\hline \multirow{4}{*}{ Months } & \multirow{4}{*}{$\begin{array}{c}\text { No. of } \\
\text { Rats }\end{array}$} & \multicolumn{4}{|c|}{ Sex } & \multicolumn{12}{|c|}{ Maturity status } \\
\hline & & \multirow{2}{*}{\multicolumn{2}{|c|}{ Males }} & \multirow{2}{*}{\multicolumn{2}{|c|}{ Females }} & \multicolumn{4}{|c|}{ Males } & \multicolumn{4}{|c|}{ Females } & \multicolumn{4}{|c|}{ Total } \\
\hline & & & & & & \multicolumn{2}{|c|}{ Immature } & \multicolumn{2}{|c|}{ Mature } & \multicolumn{2}{|c|}{ Immature } & \multicolumn{2}{|c|}{ Mature } & \multicolumn{2}{|c|}{ Immature } & \multicolumn{2}{|c|}{ Mature } \\
\hline & & No. & $\%$ & No. & $\%$ & No. & $\%$ & No. & $\%$ & No. & $\%$ & No. & $\%$ & No. & $\%$ & No. & $\%$ \\
\hline Nov.05 & 17 & 7 & 41.2 & 10 & 58.8 & 3 & 42.9 & 4 & 57.1 & 4 & 40.0 & 6 & 60.0 & & 41.2 & 10 & 58.8 \\
\hline Dec. & 19 & 9 & 47.4 & 10 & 52.6 & 0 & 0.0 & 9 & 100.0 & 3 & 30.0 & 7 & 70.0 & 3 & 15.8 & 16 & 84.2 \\
\hline Jan.06 & 15 & 5 & 33.3 & 10 & 66.7 & 3 & 60.0 & 2 & 40.0 & 3 & 30.0 & 7 & 70.0 & 6 & 40.0 & 9 & 60.0 \\
\hline Feb. & 9 & 6 & 66.7 & 3 & 33.3 & 2 & 33.3 & 4 & 66.7 & 2 & 66.7 & 1 & 33.3 & 4 & 44.4 & 5 & 55.6 \\
\hline Mar. & 9 & 7 & 77.8 & 2 & 22.2 & 2 & 28.6 & 5 & 71.4 & 0 & 0.0 & 2 & 100.0 & 2 & 22.2 & 7 & 77.8 \\
\hline Apr. & 41 & 24 & 58.5 & 17 & 41.5 & 4 & 16.7 & 20 & 83.3 & 2 & 11.8 & 15 & 88.2 & 6 & 14.6 & 35 & 85.4 \\
\hline May & 41 & 16 & 39.0 & 25 & 61.0 & 6 & 37.5 & 10 & 62.5 & 12 & 48.0 & 13 & 52.0 & 18 & 43.9 & 23 & 56.1 \\
\hline June & 37 & 23 & 62.2 & 14 & 37.8 & 2 & 8.7 & 21 & 91.3 & 3 & 21.4 & 11 & 78.6 & 5 & 13.5 & 32 & 86.5 \\
\hline July & 32 & 16 & 50.0 & 16 & 50.0 & 6 & 37.5 & 10 & 62.5 & 3 & 18.8 & 13 & 81.3 & 9 & 28.1 & 23 & 71.9 \\
\hline Aug & 56 & 28 & 50.0 & 28 & 50.0 & 15 & 53.6 & 13 & 46.4 & 10 & 35.7 & 18 & 64.3 & 25 & 44.6 & 31 & 55.4 \\
\hline Sep. & 29 & 13 & 44.8 & 16 & 55.2 & 1 & 7.7 & 12 & 92.3 & 1 & 6.3 & 15 & 93.8 & 2 & 6.9 & 27 & 93.1 \\
\hline Oct. & 24 & 8 & 33.3 & 16 & 66.7 & 1 & 12.5 & 7 & 87.5 & 2 & 12.5 & 14 & 87.5 & 3 & 12.5 & 21 & 87.5 \\
\hline Total & 329 & 162 & - & 167 & - & 45 & - & 117 & - & 45 & - & 122 & - & 90 & - & 239 & - \\
\hline$\%$ & - & - & 49.2 & - & 50.8 & - & 27.8 & - & 72.2 & - & 26.9 & - & 73.1 & - & 27.4 & - & 72.6 \\
\hline
\end{tabular}

Sex ratio (males/females) $=0.98: 1.02$ 
Table 3. Number of trapped roof rats Rattus rattus (Linn.), their sex ratio and maturity status at the granaries location during year of 2004/2005.

\begin{tabular}{|c|c|c|c|c|c|c|c|c|c|c|c|c|c|c|c|c|c|}
\hline \multirow{4}{*}{ Months } & \multirow{4}{*}{$\begin{array}{l}\text { No. } \\
\text { of } \\
\text { Rats }\end{array}$} & \multicolumn{4}{|c|}{ Sex } & \multicolumn{12}{|c|}{ Maturity status } \\
\hline & & \multirow{2}{*}{\multicolumn{2}{|c|}{ Males }} & \multirow{2}{*}{\multicolumn{2}{|c|}{ Females }} & \multicolumn{4}{|c|}{ Males } & \multicolumn{4}{|c|}{ Females } & \multicolumn{4}{|c|}{ Total } \\
\hline & & & & & & \multicolumn{2}{|c|}{ Immature } & \multicolumn{2}{|c|}{ Mature } & \multicolumn{2}{|c|}{ Immature } & \multicolumn{2}{|c|}{ Mature } & \multicolumn{2}{|c|}{ Immature } & \multicolumn{2}{|c|}{ Mature } \\
\hline & & No. & $\%$ & No. & $\%$ & No. & $\%$ & No. & $\%$ & No. & $\%$ & No. & $\%$ & No. & $\%$ & No. & $\%$ \\
\hline Nov.04 & 7 & 5 & 71.43 & 2 & 28.6 & 3 & 60.0 & 2 & 40.0 & 1 & 50.0 & 1 & 50.0 & 4 & 57.1 & 3 & 42.9 \\
\hline Dec. & 5 & 3 & 60.00 & 2 & 40.0 & 0 & 0.0 & 3 & 100.0 & 0 & 0.0 & 2 & 100.0 & 0 & 0.0 & 5 & 100.0 \\
\hline Jan.05 & 3 & 1 & 33.33 & 2 & 66.7 & 0 & 0.0 & 1 & 100.0 & 0 & 0.0 & 2 & 100.0 & 0 & 0.0 & 3 & 100.0 \\
\hline Feb. & 3 & 2 & 66.67 & 1 & 33.3 & 0 & 0.0 & 2 & 100.0 & 0 & 0.0 & 1 & 100.0 & 0 & 0.0 & 3 & 100.0 \\
\hline Mar. & 3 & 0 & 0.00 & 3 & 100.0 & 0 & 0.0 & 0 & 0.0 & 0 & 0.0 & 3 & 100.0 & 0 & 0.0 & 3 & 100.0 \\
\hline Apr. & 13 & 4 & 30.77 & 9 & 69.2 & 1 & 25.0 & 3 & 75.0 & 1 & 11.1 & 8 & 88.9 & 2 & 15.4 & 11 & 84.6 \\
\hline May & 20 & 14 & 70.00 & 6 & 30.0 & 4 & 28.6 & 10 & 71.4 & 1 & $\begin{array}{l}16.7 \\
\end{array}$ & 5 & 83.3 & 5 & 25.0 & 15 & 75.0 \\
\hline June & 1 & 0 & 0.00 & 1 & 100.0 & 0 & 0.0 & 0 & 0.0 & 0 & 0.0 & 1 & 100.0 & 0 & 0.0 & 1 & 100.0 \\
\hline July & 3 & 0 & 0.00 & 3 & 100.0 & 0 & 0.0 & 0 & 0.0 & 1 & 33.3 & 2 & 66.7 & 1 & 33.3 & 2 & 66.7 \\
\hline Aug & 10 & 5 & 50.00 & 5 & 50.0 & 1 & 20.0 & 4 & 80.0 & 0 & 0.0 & 5 & 100.0 & 1 & 10.0 & 9 & 90.0 \\
\hline Sep. & 13 & 5 & 38.46 & 8 & 61.5 & 1 & 20.0 & 4 & 80.0 & 0 & 0.0 & 8 & 100.0 & 1 & 7.7 & 12 & 92.3 \\
\hline Oct. & 3 & 1 & 33.33 & 2 & 66.7 & 0 & 0.0 & 1 & 100.0 & 0 & 0.0 & 2 & 100.0 & 0 & 0.0 & 3 & 100.0 \\
\hline Total & 84 & 40 & - & 44 & - & 10 & - & 30 & - & 4 & - & 40 & - & 14 & - & 70 & - \\
\hline$\%$ & - & - & 47.62 & - & 52.4 & - & 25.0 & - & 75.0 & - & 9.1 & - & 90.9 & - & 16.7 & - & 83.3 \\
\hline
\end{tabular}

Sex ratio (males/females) $=0.95: 1.05$ 
Table 4. Number of trapped roof rats Rattus rattus (Linn.), their sex ratio and maturity status at the granaries location during year of 2005/2006.

\begin{tabular}{|c|c|c|c|c|c|c|c|c|c|c|c|c|c|c|c|c|c|}
\hline \multirow{4}{*}{ Months } & \multirow{4}{*}{$\begin{array}{l}\text { No. of } \\
\text { Rats }\end{array}$} & \multicolumn{4}{|c|}{ Sex } & \multicolumn{12}{|c|}{ Maturity status } \\
\hline & & \multirow{2}{*}{\multicolumn{2}{|c|}{ Males }} & \multirow{2}{*}{\multicolumn{2}{|c|}{ Females }} & \multicolumn{4}{|c|}{ Males } & \multicolumn{4}{|c|}{ Females } & \multicolumn{4}{|c|}{ Total } \\
\hline & & & & & & \multicolumn{2}{|c|}{ Immature } & \multicolumn{2}{|c|}{ Mature } & \multicolumn{2}{|c|}{ Immature } & \multicolumn{2}{|c|}{ Mature } & \multicolumn{2}{|c|}{ Immature } & \multicolumn{2}{|c|}{ Mature } \\
\hline & & No. & $\%$ & No. & $\%$ & No. & $\%$ & No. & $\%$ & No. & $\%$ & No. & $\%$ & No. & $\%$ & No. & $\%$ \\
\hline Nov. 05 & 13 & 8 & 61.5 & 5 & 38.5 & 1 & 12.5 & 7 & 87.5 & 3 & 60.0 & 2 & 40.0 & 4 & 30.8 & 9 & 69.2 \\
\hline Dec. & 7 & 3 & 42.9 & 4 & 57.1 & 0 & 0.0 & 3 & 100.0 & 1 & 25.0 & 3 & 75.0 & 1 & 14.3 & 6 & 85.7 \\
\hline Jan.06 & 6 & 5 & 83.3 & 1 & 16.7 & 0 & 0.0 & 5 & 100.0 & 0 & 0.0 & 1 & 100.0 & 0 & 0.0 & 6 & 100.0 \\
\hline Feb. & 5 & 4 & 80.0 & 1 & 20.0 & 0 & 0.0 & 4 & 100.0 & 0 & 0.0 & 1 & 100.0 & 0 & 0.0 & 5 & 100.0 \\
\hline Mar. & 0 & 0 & 0.0 & 0 & 0.0 & 0 & 0.0 & 0 & 0.0 & 0 & 0.0 & 0 & 0.0 & 0 & 0.0 & 0 & 0.0 \\
\hline Apr. & 2 & 2 & 100.0 & 0 & 0.0 & 0 & 0.0 & 2 & 100.0 & 0 & 0.0 & 0 & 0.0 & 0 & 0.0 & 2 & 100.0 \\
\hline May & 9 & 7 & 77.8 & 2 & 22.2 & 0 & 0.0 & 7 & 100.0 & 0 & 0.0 & 2 & 100.0 & 0 & 0.0 & 9 & 100.0 \\
\hline June & 0 & 0 & 0.0 & 0 & 0.0 & 0 & 0.0 & 0 & 0.0 & 0 & 0.0 & 0 & 0.0 & 0 & 0.0 & 0 & 0.0 \\
\hline July & 3 & 3 & 100.0 & 0 & 0.0 & 0 & 0.0 & 3 & 100.0 & 0 & 0.0 & 0 & 0.0 & 0 & 0.0 & 3 & 100.0 \\
\hline Aug & 7 & 3 & 42.9 & 4 & 57.1 & 0 & 0.0 & 3 & 100.0 & 2 & 50.0 & 2 & 50.0 & 2 & 28.6 & 5 & 71.4 \\
\hline Sep. & 3 & 3 & 100.0 & 0 & 0.0 & 0 & 0.0 & 3 & 100.0 & 0 & 0.0 & 0 & 0.0 & 0 & 0.0 & 3 & 100.0 \\
\hline Oct. & 12 & 8 & 66.7 & 4 & 33.3 & 1 & 12.5 & 7 & 87.5 & 2 & 50.0 & 2 & 50.0 & 3 & 25.0 & 9 & 75.0 \\
\hline Total & 67 & 46 & - & 21 & - & 2 & - & 44 & - & 8 & - & 13 & - & 10 & - & 57 & - \\
\hline$\%$ & - & - & 68.7 & - & 31.3 & - & 4.3 & - & 95.7 & - & 38.1 & - & 61.9 & - & 14.9 & - & 85.1 \\
\hline
\end{tabular}

Sex ratio $($ males/females $)=1.37: 0.63$ 
Table 5. Number of trapped roof rats Rattus rattus (Linn.), their sex ratio and maturity status at the poultry farm location during year of $2004 / 2005$.

\begin{tabular}{|c|c|c|c|c|c|c|c|c|c|c|c|c|c|c|c|c|c|}
\hline \multirow{4}{*}{ Months } & \multirow{4}{*}{$\begin{array}{l}\text { No. } \\
\text { of } \\
\text { Rats }\end{array}$} & \multicolumn{4}{|c|}{ Sex } & \multicolumn{12}{|c|}{ Maturity status } \\
\hline & & \multirow{2}{*}{\multicolumn{2}{|c|}{ Males }} & \multirow{2}{*}{\multicolumn{2}{|c|}{ Females }} & \multicolumn{4}{|c|}{ Males } & \multicolumn{4}{|c|}{ Females } & \multicolumn{4}{|c|}{ Total } \\
\hline & & & & & & \multicolumn{2}{|c|}{ Immature } & \multicolumn{2}{|c|}{ Mature } & \multicolumn{2}{|c|}{ Immature } & \multicolumn{2}{|c|}{ Mature } & \multicolumn{2}{|c|}{ Immature } & \multicolumn{2}{|c|}{ Mature } \\
\hline & & No. & $\%$ & No. & $\%$ & No. & $\%$ & No. & $\%$ & No. & $\%$ & No. & $\%$ & No. & $\%$ & No. & $\%$ \\
\hline Nov.04 & 5 & 4 & 80.0 & 1 & 20.0 & 2 & 50.0 & 2 & 50.0 & 0 & 0.0 & 1 & 100.0 & 2 & 40.0 & 3 & 60.0 \\
\hline Dec. & 5 & 1 & 20.0 & 4 & 80.0 & 0 & 0.0 & 1 & 100.0 & 2 & 50.0 & 2 & 50.0 & 2 & 40.0 & 3 & 60.0 \\
\hline Jan.05 & 7 & 4 & 57.1 & 3 & 42.9 & 2 & 50.0 & 2 & 50.0 & 0 & 0.0 & 3 & 100.0 & 2 & 28.6 & 5 & 71.4 \\
\hline Feb. & 3 & 1 & 33.3 & 2 & 66.7 & 0 & 0.0 & 1 & 100.0 & 1 & 50.0 & 1 & 50.0 & 1 & 33.3 & 2 & 66.7 \\
\hline Mar. & 6 & 3 & 50.0 & 3 & 50.0 & 2 & 66.7 & 1 & 33.3 & 0 & 0.0 & 3 & 100.0 & 2 & 33.3 & 4 & 66.7 \\
\hline Apr. & 6 & 4 & 66.7 & 2 & 33.3 & 0 & 0.0 & 4 & 100.0 & 0 & 0.0 & 2 & 100.0 & 0 & 0.0 & 6 & 100.0 \\
\hline May & 15 & 4 & 26.7 & 11 & 73.3 & 2 & 50.0 & 2 & 50.0 & 0 & 0.0 & 11 & 100.0 & 2 & 13.3 & 13 & 86.7 \\
\hline June & 10 & 3 & 30.0 & 7 & 70.0 & 1 & 33.3 & 2 & 66.7 & 2 & 28.6 & 5 & 71.4 & 3 & 30.0 & 7 & 70.0 \\
\hline July & 2 & 0 & 0.0 & 2 & 100.0 & 0 & 0.0 & 0 & 0.0 & 0 & 0.0 & 2 & 100.0 & 0 & 0.0 & 2 & 100.0 \\
\hline Aug & 11 & 3 & 27.3 & 8 & 72.7 & 2 & 66.7 & 1 & 33.3 & 3 & 37.5 & 5 & 62.5 & 5 & 45.5 & 6 & 54.5 \\
\hline Sep. & 10 & 5 & 50.0 & 5 & 50.0 & 2 & 40.0 & 3 & 60.0 & 0 & 0.0 & 5 & 100.0 & 2 & 20.0 & 8 & 80.0 \\
\hline Oct. & 6 & 3 & 50.0 & 3 & 50.0 & 0 & 0.0 & 3 & 100.0 & 0 & 0.0 & 3 & 100.0 & 0 & 0.0 & 6 & 100.0 \\
\hline Total & 86 & 35 & - & 51 & - & 13 & - & 22 & - & 8 & - & 43 & - & 21 & - & 65 & - \\
\hline$\%$ & - & - & 40.7 & - & 59.3 & - & 37.1 & - & 62.9 & - & 15.7 & - & 84.3 & - & 24.4 & - & 75.6 \\
\hline
\end{tabular}

Sex ratio (males/females) $=0.81: 1.19$ 
Table 6. Number of trapped roof rats Rattus rattus (Linn.), their sex ratio and maturity status at the poultry farm location during year of $2005 / 2006$.

\begin{tabular}{|c|c|c|c|c|c|c|c|c|c|c|c|c|c|c|c|c|c|}
\hline \multirow{4}{*}{ Months } & \multirow{4}{*}{$\begin{array}{l}\text { No. of } \\
\text { Rats }\end{array}$} & \multicolumn{4}{|c|}{ Sex } & \multicolumn{12}{|c|}{ Maturity status } \\
\hline & & \multirow{2}{*}{\multicolumn{2}{|c|}{ Males }} & \multirow{2}{*}{\multicolumn{2}{|c|}{ Females }} & \multicolumn{4}{|c|}{ Males } & \multicolumn{4}{|c|}{ Females } & \multicolumn{4}{|c|}{ Total } \\
\hline & & & & & & \multicolumn{2}{|c|}{ Immature } & \multicolumn{2}{|c|}{ Mature } & \multicolumn{2}{|c|}{ Immature } & \multicolumn{2}{|c|}{ Mature } & \multicolumn{2}{|c|}{ Immature } & \multicolumn{2}{|c|}{ Mature } \\
\hline & & No. & $\%$ & No. & $\%$ & No. & $\%$ & No. & $\%$ & No. & $\%$ & No. & $\%$ & No. & $\%$ & No. & $\%$ \\
\hline Nov.05 & 5 & 0 & 0.0 & 5 & 100.0 & 0 & 0.0 & 0 & 0.0 & 0 & 0.0 & 5 & 100.0 & 0 & 0.0 & 5 & 100.0 \\
\hline Dec. & 11 & 7 & 63.6 & 4 & 36.4 & 5 & 71.4 & 2 & 28.6 & 3 & 75.0 & 1 & 25.0 & 8 & 72.7 & 3 & 27.3 \\
\hline Jan.06 & 7 & 5 & 71.4 & 2 & 28.6 & 2 & 40.0 & 3 & 60.0 & 0 & 0.0 & 2 & 100.0 & 2 & 28.6 & 5 & 71.4 \\
\hline Feb. & 6 & 5 & 83.3 & 1 & 16.7 & 0 & 0.0 & 5 & 100.0 & 0 & 0.0 & 1 & 100.0 & 0 & 0.0 & 6 & 100.0 \\
\hline Mar. & 8 & 3 & 37.5 & 5 & 62.5 & 1 & 33.3 & 2 & 66.7 & 2 & 40.0 & 3 & 60.0 & 3 & 37.5 & 5 & 62.5 \\
\hline Apr. & 8 & 3 & 37.5 & 5 & 62.5 & 0 & 0.0 & 3 & 100.0 & 4 & 80.0 & 1 & 20.0 & 4 & 50.0 & 4 & 50.0 \\
\hline May & 17 & 4 & 23.5 & 13 & 76.5 & 2 & 50.0 & 2 & 50.0 & 8 & 61.5 & 5 & 38.5 & 10 & 58.8 & 7 & 41.2 \\
\hline June & 3 & 1 & 33.3 & 2 & 66.7 & 1 & 100.0 & 0 & 0.0 & 2 & 100.0 & 0 & 0.0 & 3 & 100.0 & 0 & 0.0 \\
\hline July & 1 & 1 & 100.0 & 0 & 0.0 & 0 & 0.0 & 1 & 100.0 & 0 & 0.0 & 0 & 0.0 & 0 & 0.0 & 1 & 100.0 \\
\hline Aug & 0 & 0 & 0.0 & 0 & 0.0 & 0 & 0.0 & 0 & 0.0 & 0 & 0.0 & 0 & 0.0 & 0 & 0.0 & 0 & 0.0 \\
\hline Sep. & 0 & 0 & 0.0 & 0 & 0.0 & 0 & 0.0 & 0 & 0.0 & 0 & 0.0 & 0 & 0.0 & 0 & 0.0 & 0 & 0.0 \\
\hline Oct. & 0 & 0 & 0.0 & 0 & 0.0 & 0 & 0.0 & 0 & 0.0 & 0 & 0.0 & 0 & 0.0 & 0 & 0.0 & 0 & 0.0 \\
\hline Total & 66 & 29 & - & 37 & - & 11 & - & 18 & - & 19 & - & 18 & - & 30 & - & 36 & - \\
\hline$\%$ & - & - & 43.9 & - & 56.1 & - & 37.9 & - & 62.1 & - & 51.4 & - & 48.6 & - & 45.5 & - & 54.5 \\
\hline
\end{tabular}

Sex ratio (males/females) $=0.88: 1.12$ 
Table 7. Average number of embryos per pregnant female for the roof rats Rattus rattus (Linn.) trapped from three locations during year of 2004/2005.

\begin{tabular}{|c|c|c|c|c|c|c|c|c|c|c|c|c|c|c|c|c|}
\hline \multirow[b]{2}{*}{ Months } & \multicolumn{4}{|c|}{ Houses } & \multicolumn{4}{|c|}{ Granaries } & \multicolumn{4}{|c|}{ Poultry farm } & \multicolumn{4}{|c|}{ Total } \\
\hline & $\begin{array}{l}\text { No. of } \\
\text { Preg. F. }\end{array}$ & $\begin{array}{l}\text { No. of } \\
\text { Non } \\
\text { Preg. F. }\end{array}$ & $\begin{array}{l}\text { T. No. } \\
\text { of } E \text {. }\end{array}$ & $\begin{array}{l}\text { M. No. } \\
\text { of } E \text {. }\end{array}$ & $\begin{array}{l}\text { No. of } \\
\text { Preg. F. }\end{array}$ & $\begin{array}{c}\text { No. of } \\
\text { Non } \\
\text { Preg. F. }\end{array}$ & $\begin{array}{l}\text { T. No. } \\
\text { of } E \text {. }\end{array}$ & $\begin{array}{l}\text { M. } \\
\text { No. of } \\
\text { E. }\end{array}$ & $\begin{array}{l}\text { No. of } \\
\text { Preg. F. }\end{array}$ & $\begin{array}{c}\text { No. of } \\
\text { Non } \\
\text { Preg. F. }\end{array}$ & $\begin{array}{l}\text { T. No. } \\
\text { of } E \text {. }\end{array}$ & $\begin{array}{c}\text { M. } \\
\text { No. of } \\
\text { E. }\end{array}$ & $\begin{array}{l}\text { No. of } \\
\text { Preg. F. }\end{array}$ & $\begin{array}{c}\text { No. of } \\
\text { Non } \\
\text { Preg. F. }\end{array}$ & $\begin{array}{l}\text { T. No. } \\
\text { of } E \text {. }\end{array}$ & $\begin{array}{l}\text { M. } \\
\text { No. of } \\
\text { E. }\end{array}$ \\
\hline Nov.04 & 2 & 3 & 16 & 8.0 & 0 & 1 & 0 & 0.0 & 0 & 1 & 0 & 0.0 & 2 & 5 & 16 & 8.0 \\
\hline Dec. & 3 & 2 & 17 & 5.7 & 0 & 2 & 0 & 0.0 & 0 & 2 & 0 & 0.0 & 3 & 6 & 17 & 5.7 \\
\hline Jan.05 & 3 & 4 & 16 & 5.3 & 0 & 2 & 0 & 0.0 & 2 & 1 & 12 & 6.0 & 5 & 7 & 28 & 5.6 \\
\hline Feb. & 5 & 8 & 40 & 8.0 & 0 & 1 & 0 & 0.0 & 0 & 1 & 0 & 0.0 & 5 & 10 & 40 & 8.0 \\
\hline Mar. & 1 & 2 & 11 & 11.0 & 1 & 2 & 5 & 5.0 & 0 & 3 & 0 & 0.0 & 2 & 7 & 16 & 8.0 \\
\hline Apr. & 12 & 11 & 92 & 7.7 & 2 & 6 & 12 & 6.0 & 2 & 0 & 16 & 8.0 & 16 & 17 & 120 & 7.5 \\
\hline May & 10 & 16 & 57 & 5.7 & 2 & 3 & 16 & 8.0 & 5 & 6 & 40 & 8.0 & 17 & 25 & 113 & 6.6 \\
\hline June & 11 & 5 & 80 & 7.3 & 0 & 1 & 0 & 0.0 & 5 & 0 & 33 & 6.6 & 16 & 6 & 113 & 7.1 \\
\hline July & 1 & 9 & 6 & 6.0 & 0 & 2 & 0 & 0.0 & 0 & 2 & 0 & 0.0 & 1 & 13 & 6 & 6.0 \\
\hline Aug & 8 & 11 & 48 & 6.0 & 1 & 4 & 7 & 7.0 & 0 & 5 & 0 & 0.0 & 9 & 20 & 55 & 6.1 \\
\hline Sep. & 9 & 9 & 57 & 6.3 & 2 & 6 & 15 & 7.5 & 2 & 3 & 19 & 9.5 & 13 & 18 & 91 & 7.0 \\
\hline Oct. & 4 & 6 & 32 & 8.0 & 0 & 2 & 0 & 0.0 & 3 & 0 & 15 & 5.0 & 7 & 8 & 47 & 6.7 \\
\hline Total & 69 & 86 & 472 & & 8 & 32 & 55 & & 19 & 24 & 135 & & 96 & 142 & 662 & \\
\hline Mean & & & & 6.8 & & & & 6.9 & & & & 7.1 & & & & 6.9 \\
\hline
\end{tabular}


Table 8. Average number of embryos per pregnant female for the roof rats Rattus rattus (Linn.) trapped from three locations during year of 2004/2005.

\begin{tabular}{|c|c|c|c|c|c|c|c|c|c|c|c|c|c|c|c|c|}
\hline \multirow[b]{2}{*}{ Months } & \multicolumn{4}{|c|}{ Houses } & \multicolumn{4}{|c|}{ Granaries } & \multicolumn{4}{|c|}{ Poultry farm } & \multicolumn{4}{|c|}{ Total } \\
\hline & $\begin{array}{l}\text { No. of } \\
\text { Preg. F. }\end{array}$ & $\begin{array}{l}\text { No. of } \\
\text { Non } \\
\text { Preg. F. }\end{array}$ & $\begin{array}{l}\text { T. No. } \\
\text { of } E \text {. }\end{array}$ & $\begin{array}{l}\text { M. No. } \\
\text { of } E \text {. }\end{array}$ & $\begin{array}{l}\text { No. of } \\
\text { Preg. F. }\end{array}$ & $\begin{array}{l}\text { No. of } \\
\text { Non } \\
\text { Preg. F. }\end{array}$ & $\begin{array}{l}\text { T. No. } \\
\text { of } E \text {. }\end{array}$ & $\begin{array}{l}\text { M. No. } \\
\text { of } E \text {. }\end{array}$ & $\begin{array}{l}\text { No. of } \\
\text { Preg. F. }\end{array}$ & $\begin{array}{l}\text { No. of } \\
\text { Non } \\
\text { Preg. F. }\end{array}$ & $\begin{array}{l}\text { T. No. } \\
\text { of } E \text {. }\end{array}$ & $\begin{array}{l}\text { M. No. } \\
\text { of E. }\end{array}$ & $\begin{array}{l}\text { No. of } \\
\text { Preg. F. }\end{array}$ & $\begin{array}{l}\text { No. of } \\
\text { Non } \\
\text { Preg. F. }\end{array}$ & $\begin{array}{l}\text { T. No. } \\
\text { of } E \text {. }\end{array}$ & $\begin{array}{c}M . \\
\text { No. } \\
\text { of } E .\end{array}$ \\
\hline Nov.05 & 0 & 6 & 0 & 0.0 & 0 & 2 & 0 & 0.0 & 3 & 2 & 23 & 7.7 & 3 & 10 & 23 & 7.7 \\
\hline Dec. & 3 & 4 & 20 & 6.7 & 0 & 3 & 0 & 0.0 & 1 & 0 & 6 & 6.0 & 4 & 7 & 26 & 6.5 \\
\hline Jan.06 & 2 & 5 & 16 & 8.0 & 0 & 1 & 0 & 0.0 & 0 & 2 & 0 & 0.0 & 2 & 8 & 16 & 8.0 \\
\hline Feb. & 0 & 1 & 0 & 0.0 & 0 & 1 & 0 & 0.0 & 0 & 1 & 0 & 0.0 & 0 & 3 & 0 & 0.0 \\
\hline Mar. & 0 & 2 & 0 & 0.0 & 0 & 0 & 0 & 0.0 & 1 & 2 & 5 & 5.0 & 1 & 4 & 5 & 5.0 \\
\hline Apr. & 3 & 12 & 19 & 6.3 & 0 & 0 & 0 & 0.0 & 0 & 1 & 0 & 0.0 & 3 & 13 & 19 & 6.3 \\
\hline May & 3 & 10 & 20 & 6.7 & 1 & 1 & 6 & 6.0 & 4 & 1 & 32 & 8.0 & 8 & 12 & 58 & 7.3 \\
\hline June & 1 & 10 & 7 & 7.0 & 0 & 0 & 0 & 0.0 & 0 & 0 & 0 & 0.0 & 1 & 10 & 7 & 7.0 \\
\hline July & 3 & 10 & 19 & 6.3 & 0 & 0 & 0 & 0.0 & 0 & 0 & 0 & 0.0 & 3 & 10 & 19 & 6.3 \\
\hline Aug & 3 & 15 & 20 & 6.7 & 0 & 2 & 0 & 0.0 & 0 & 0 & 0 & 0.0 & 3 & 17 & 20 & 6.7 \\
\hline Sep. & 2 & 13 & 12 & 6.0 & 0 & 0 & 0 & 0.0 & 0 & 0 & 0 & 0.0 & 2 & 13 & 12 & 6.0 \\
\hline Oct. & 6 & 8 & 40 & 6.7 & 0 & 0 & 0 & 0.0 & 0 & 0 & 0 & 0.0 & 6 & 8 & 40 & 6.7 \\
\hline Total & 26 & 96 & 173 & & 1 & 10 & 6 & & 9 & 9 & 66 & & 36 & 115 & 245 & \\
\hline Mean & & & & 6.7 & & & & 6.0 & & & & 7.3 & & & & 6.8 \\
\hline
\end{tabular}

No. of Preg. F.: Number of pregnant female; No. of Non Preg. F.: Number of non pregnant female T. No. of E.: Total number of embryos; M. No. of E.: Mean number of embryos per pregnant female 


\section{REFERENCES}

1. Abdel-Gawad, K. H. 1974. Ecological and toxicological studies on commensal and household rodents in Assiut area. M.Sc. Thesis, Fac. of Agric., Assiut Univ.

2. Abdel-Gawad, K. H. 1979. Studies on the interrelation between rodents and their ectoparasites in the cultivated and semi-arid zones. Ph.D. Thesis, Fac. of Agric., Assiut Univ., pp 141.

3. Abdel-Gawad, K. H., A. M. Salit, T. Y. Helal, A. Maher-Ali and M. S. Arafa. 1982. Sex ratio and maturity status of rodent species in semi-arid newly reclaimed land in eastern desert. Assiut. J. Agric. Sci., 13 (2): 3-10.

4. Abd-El-Karim, S. M. 1991. Studies on rodents in Sharkia Governorate. Ph.D. Thesis, Fac. of Agric., Zagazig Univ., pp. 294.

5. Arafa, M. S. 1968. Studies on ecto-and endoparasites of rats and mice in A.R.E. with special reference to parasites potentially transmissible to man. M.D. Thesis, Parasit., Fac. Med., Ain-Shams Univ. Cairo, A.R.E.

6. Dykstra, W. W. 1966. The economic important of commensal rodents. WHO/Vector control, 66-217.

7. El-Bahrawy, A. A. F. 1986. Studies on ecology and control of some rodent species in Ismalia Governorate. Ph.D. Thesis, Fac. of Agric. Suez Canal, Univ., pp. 235.

8. El-Bahrawy, A. A. F., A. M. I. Hegab, G. A. El-Kady and M. I. A. Elbkhshwangi. 2008. Field studies on commensal rodents at El-Ibrahemia District, Sharkia Governorate. Egypt J. Appl. Sci., 23 (5): 230-243.

9. Embarak, M. Z. 1997. Ecological and control studies on rodents and their ectoparasites in cultivated and newly reclaimed areas. M. Sc. Thesis, Fac. Agric. Assiut Univ., pp. 130.

10. Gaaboub, I. A., A. E. E. Widaatalla and M. F. El-Sawy. 1978. Seasonal occurrence of small rodents in relation to cultivated area in the vicinity of Alexandria Governorate, Egypt. J. Agric. Res. Tanta. Univ., 4 (2): 312-322.

11. Hegab, A. M. I. 2004. Studies on some commensal rodent species and their ectoparsites in different locations at Sharkia Governorate. Agric. Res. J. Suez Canal Univ., 4 (2): 119-124. 
12. Hegab, A. M. I., Sh. A. A. Ismail and S. A. A. El-Massry. 2006. Survey, morphological studies and ectoparsites associated with some commensal rat species at Sharkia Governorate. Egypt. J. of Appl. Sci., 21 (5): 340-349.

13. Mourad, M. G., K. H. Abdel-Gawad and A. Maher-Ali. 1982. Population density of rodent species in some urban area in Minia Governorate. Assiut J. Agric. Sci., 13 (2): $19-25$.

14. Omar, M. T. 1977. Studies on rodents attacking stores and food staff factories in Cairo. Ph.D. Thesis, Fac. Agric., AL-Zahra Univ. Cairo, pp. 150.

15. Osbron, D. J. and I. Helmy. 1980. The contemporary land mammals of Egypt (including Sinai). Published by Field Museum of National History. London.

16. Youssef, A. E. S. 1996. Ecological, biological and toxicological studies on rats in stores and shounas. Ph. D. Thesis, Agric., Minoufia Univ., pp. 132. 


\section{حصر لجرذ الأسقف Rattus rattus (Linn.) في مركز الإبراهيمية}

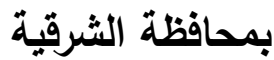

أحمد مصطفي إبراهيم حجاب' ، عوض عبد الله فرحات البحراوي` ، جمال عبد الستار القاضي ' ،

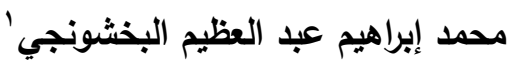

$$
\begin{aligned}
& 1 \text { - معهد بحوث وقاية النباتات - مركز البحوث الزراعبة - الدقى - الجيزة } \\
& \text { r - قسم وقاية النبات - كلية الزراعة - جامعة قناة السوبيس }
\end{aligned}
$$

تم إجراء حصر لجرذ الأسقف Rattus rattus (Linn.) بواسطة المصائد الحية في ثناث أماكن مختلفة هي (المنازل ومخازن الحبوب ومزرعة دواجن) في مركز الإبراهيمية بمحافظة الثرقية

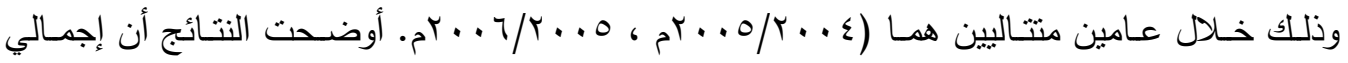

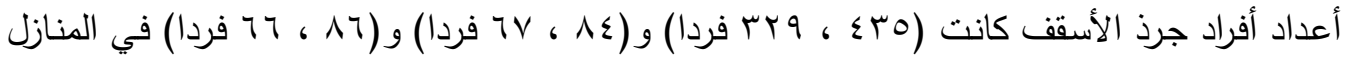

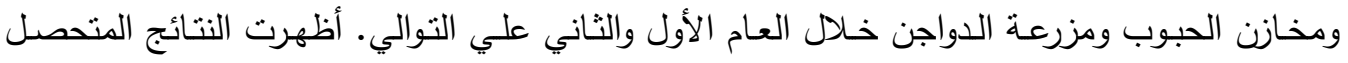

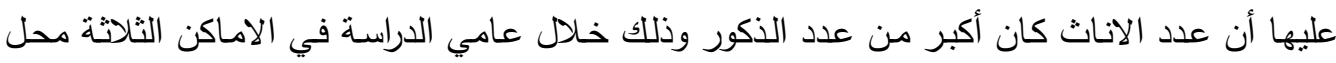

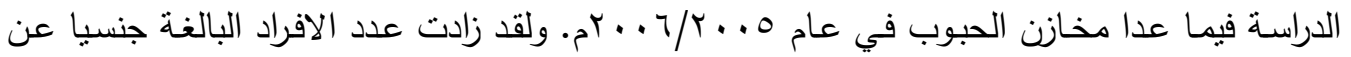

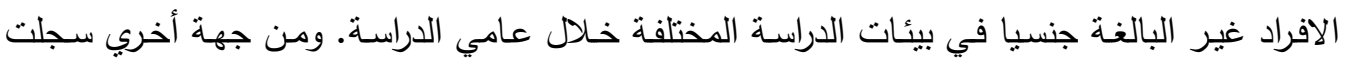

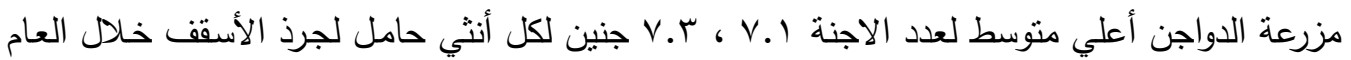
الاول والثاني علي التوالي. 